\title{
Ocorrência e morfologia de glândulas tegumentares no abdome de Melissoptila richardiae (Bertoni \& Scrottky) (Hymenoptera, Apidae, Eucerini)
}

\author{
Carminda da Cruz-Landim ${ }^{1} \&$ Vagner T. Paes de Oliveira ${ }^{1,2}$
}

\begin{abstract}
${ }^{1}$ Departamento de Biologia, Instituto de Biociências, Universidade Estadual Paulista. Av. 24A, n ${ }^{\circ}$ 1515, Bela Vista, Rio Claro-SP, $13506-900$. cclandim@rc.unesp.br

${ }^{2}$ Bolsista FAPESP - Proc. 97/09644-3. vagnertadeu@yahoo.com.br
\end{abstract}

\begin{abstract}
Occurrence and morphology of tegumentary abdominal glands in Melissoptila richardiae (Bertoni \& Scrottky) (Hymenoptera, Apidae, Eucerini). The occurrence and morphology of abdominal tegumentary glands were studied in Melissoptila richardiae. The results showed the existence of class III glandular cells that may appear as isolated units in tergites and sternites III and IV or forming clustered cells in both side of the tergites III and IV. In this late case the secretion is released in reservoirs derived from the tergite intersegmental membranes. The results suggest that this last gland products are lipidic and probably volatile.
\end{abstract}

KEYWORDS. Abdominal glands, esternites, reservoir, tergites, volatiles.

RESUMO. Ocorrência e morfologia de glândulas tegumentares no abdome de Melissoptila richardiae (Bertoni \& Scrottky) (Hymenoptera, Apidae, Eucerini). Foram estudadas a ocorrência e a morfologia de glândulas tegumentares presentes no abdome de fêmeas de Melissoptila richardiae. Os resultados mostram que nesta espécie, células glandulares da classe III são encontradas de duas formas: isoladas nos tergitos e esternitos III e IV e formando um aglomerado de unidades glandulares bilateralmente, entre os segmentos III e IV, os quais liberam seu produto de secreção em um reservatório originado a partir da membrana intersegmental. Os resultados sugerem que o produto secretado é lipídico e, provavelmente volátil.

PALAVRAS-CHAVE. Esternitos, glândulas abdominais, reservatório, tergitos, voláteis.

Muitos trabalhos têm relatado, desde há muito tempo, a presença, localização e as características morfológicas, fisiológicas e químicas de glândulas tegumentares em abelhas (Dreyling 1903; Cruz-Landim 1963; 1967; 1992; Youssef 1975; Cruz-Landim et al. 1980a, b; Mota \& Cruz-Landim 1988; Cunha et al. 1990; Cruz-Landim \& Mota 1990; 1993; Cassier \& Lensky 1995; Cruz-Landim 1996; Cruz-Landim 1998; Salles \& CruzLandim 1998; Cruz-Landim \& Reginato 1999; Guerino \& CruzLandim 1999; 2002; 2003a; b; Cavalcante et al. 2000; Paes de Oliveira \& Cruz-Landim; 2001; Guerino \& Paes de Oliveira 2002; Ramos et al. 2004; Santos et al. 2004).

Pelo fato dessas glândulas serem órgãos especializados e diretamente relacionados com a adaptação das abelhas ao meio, estes estudos procuraram traçar, através desses caracteres, as relações entre as espécies solitárias, sociais e altamente sociais, já que muitas dessas glândulas parecem ser produtoras de substâncias voláteis que funcionam como feromônios (Wilson 1963; Velthuis 1970; Blum \& Brand 1972; Billen et al. 1986) os quais atuariam nos processos de integração social, acasalamento, delimitação de território e localização do ninho.

As glândulas tegumentares ou dermais são derivadas da epiderme do tegumento, por onde eliminam suas secreções. Noirot \& Quennedey $(1974 ; 1991)$ propuseram uma classificação para os tipos de células glandulares encontradas nos insetos, segundo a qual podem ser constituídas por três classes distintas de células, quando considerada a sua relação com a cutícula. Porém outros critérios vêm sendo adotados para a classificação dessas estruturas, como a ontogenia e função. Em abelhas têm sido encontradas glândulas formadas por células das classes I e III, estando ausentes as da classe II.

Eucerini é uma tribo de abelhas da família Apidae, com distribuição em todos os continentes exceto na Austrália (Silveira et al. 2002). Melissoptila richardiae, é uma das espécies que compõe a tribo, da qual pouco se sabe, além do fato de ser solitária e não parasita.

\section{MATERIAL E MÉTODOS}

Fêmeas adultas de Melissoptila richardiae foram obtidas em flores no campus da Universidade Estadual Paulista, em Rio Claro, São Paulo. Os esternitos e tergitos do abdômen foram fixados, separadamente, em paraformaldeído a $4 \%$, desidratados em álcool etílico, embebidos e incluídos em resina plástica Leica, segundo os procedimentos rotineiros para preparações histológicas. Cortes de $6 \mathrm{~mm}$ de espessura foram corados com Hematoxilina e Eosina, examinados e fotografados com microscopia de luz.

\section{RESULTADOS E DISCUSSÃO}

Os resultados mostraram que as fêmeas adultas de 

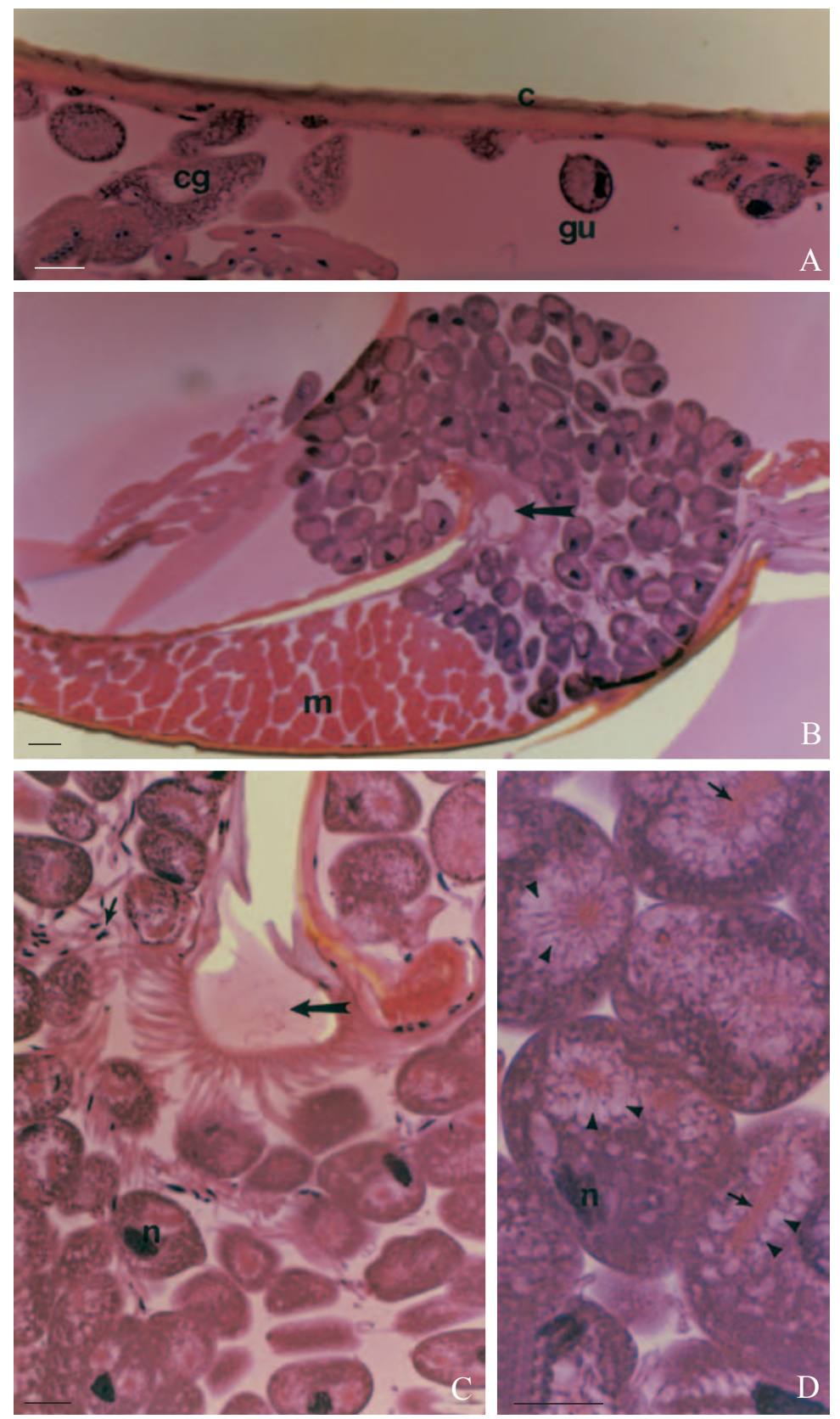

Fig. 1 - Glândulas tegumentares encontradas no abdômen de fêmea de Melissoptila richardiae. A) Células glandulares (gu) da classe III isoladas no tergito IV. cg = corpo gorduroso; $\mathrm{c}=$ cutícula; barra $=30 \mu \mathrm{m}$. B) Glândulas do tergito III com desembocadura dos canalículos, em reservatório da membrana intersegmental (seta); $\mathrm{m}=$ músculo, barra $=30 \mu \mathrm{m}$ C) Detalhe da região da desembocadura, mostrando estreitamento cuticular que forma um duto excretor. A seta maior mostra material filamentoso basófilo. A seta menor indica o núcleo do canalículo, barra $=10 \mu \mathrm{m}$. D) Células glandulares, classe III, mostrando o canalículo intracelular (seta) com conteúdo acidófilo e regiões vacuolizadas ao redor destes (cabeça de setas), $\mathrm{n}=$ núcleo, barra $=10 \mu \mathrm{m}$.

Melissoptila richardiae apresentam glândulas tegumentares constituídas por células glandulares da classe III segundo a classificação de Noirot \& Quennedey $(1974,1991)$, dorsal (nos tergitos) e ventralmente (nos esternitos) nos segmentos III e IV. Essas glândulas aparecem de duas formas: 1) como unidades secretoras isoladas distribuídas por todo esclerito (Fig. 1A) ou 2) sob a forma de agrupamentos bilaterais de células, formando cachos, com desembocadura em evaginações da membrana intersegmental que formam um reservatório (Fig. 1B).

Os cachos são formados por muitas unidades secretoras

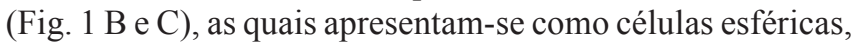
contendo núcleo condensado com vários nucléolos e citoplasma contendo o canalículo coletor. O citoplasma apresenta baixa basofilia e ao redor do canalículo coletor apresenta vacúolos com conteúdo acidófilo (Fig. 1D) ou que 
aparentam estar vazios (Fig. 1C e D). Em todos os casos o conteúdo do canalículo é acidófilo.

O fato de o citoplasma apresentar baixa basofilia e de alguns vacúolos se apresentarem vazios sugere que seu conteúdo provavelmente foi parcialmente retirado pelo processo de preparação dos tecidos sendo constituído por substâncias solúveis em álcool.

Os canalículos extracelulares das unidades secretoras se agregam e desembocam no reservatório da membrana intersegmental entre os segmentos III e IV. A secreção, que aparece intensamente acidófila na luz dos canalículos excretores, acumula-se no reservatório onde parece apresentar um componente amorfo acidófilo e outro filiforme, basófilo (Fig. 1C).

A bolsa-reservatório formada pela membrana intersegmental tem logo a seguir à região de descarga dos canalículos excretores uma dobra ou protuberância cuticular que promove um estreitamento da via de saída de secreção (Fig. 1B e C). A acumulação de secreção em reservatórios formados pela membrana intersegmental foi observada nas glândulas abdominais de Oxaea flavescens (Klug 1807) (Guerino \& Cruz-Landim 1999).

A esses tipos de glândulas unicelulares, tem sido associada à produção de substâncias com função feromonal. As substâncias melhor qualificadas para esta função são as voláteis, as quais geralmente são constituídas por hidrocarbonetos, ésteres, aldeídos e álcoois, e a ultra-estrutura dessas células mostra um retículo endoplasmático liso bastante desenvolvido e acúmulos de substâncias de aspecto lipídico no citoplasma, como observado por Mota \& Cruz-Landim (1988) e Cruz-Landim (1996). No entanto, a presença de retículo endoplasmático granular e de secreção protéica também tem sido observada nas células (Guerino \& Cruz-Landim 1999; 2002; 2003a; 2003b).

Várias das glândulas tegumentares abdominais das abelhas são sabidamente produtoras de feromônios. Às glândulas de Koschewnikow das operárias de Apis mellifera (Linaeus, 1758) é atribuída a produção de feromônios de alarme (Ghent \& Gary 1962; Maschwitz 1964) e às de rainhas, feromônios sexuais (Gary 1963; Buther \& Simpson 1965). Também as glândulas de Nassanov produzem feromônios de reconhecimento dos indivíduos da colônia (McIndoo 1914; Jacob 1924) e recentemente Espelie et al. (1990) caracterizaram a composição química do principal componente das glândulas dos tergitos de rainhas de Apis mellifera.

A função feromonal é mais freqüentemente explorada nas espécies sociais. No entanto, a comunicação química não é importante somente para estas espécies, mas também para interações intra-específicas de outra natureza, principalmente entre os sexos e até interespecíficas.

Glândulas tegumentares, que estão presentes em espécies sociais, também aparecem nas espécies solitárias de Halictidae, Oxaeinae e outras espécies de Apidae (Guerino \& Cruz-Landim 1999; 2002; 2003a) muitas vezes com a mesma localização. As glândulas de Nassanov apresentam a eliminação de sua secreção na membrana intersegmental entre os tergitos VI e
VII. Em Cephalurgus anomalus essa característica também foi observada (Cunha et al. 1990). Nos Bombini, primitivamente eussociais, a quantidade de glândulas tegumentares é muito grande e varia com as castas, sexos e tamanho das operárias, mostrando relação com a função de cada classe de indivíduo componente da colônia (Cruz-Landim 1967; Guerino \& CruzLandim 2003b). Os resultados obtidos, ainda que prévios, indicam que em Melissoptila richardiae ocorrem glândulas tegumentares que, pelas características histológicas e comparações com a literatura, podem ser produtoras de feromônios. Estudos mais detalhados precisam ser realizados, inclusive sobre o comportamento dessas abelhas, para se esclarecer a composição química e função dessas glândulas.

A localização das células isoladas permite atribuir-lhe função na produção de constituintes cuticulares. Chapman (1998) atribuiu a essas células a produção de cemento. No caso dos agrupamentos celulares que formam as glândulas dos terceiro e quarto tergitos, o fato de conterem um reservatório, sugere que seu conteúdo não seja utilizado de maneira contínua, mas armazenado para funções determinadas em condições comportamentais e temporais especiais.

\section{REFERÊNCIAS}

Billen J. P. J.; K. T. M. Dumortier \& H. H. W. Velthuis. 1986. Plasticity of honeybee castes: Occurrence of tergal glands in workers. Naturwissenschaften 73: $332-333$.

Blum, M. S. \& J. N. Brand. 1972. Social insect pheromones: their chemistry and function. American Zoology 12: 553-576.

Butler, C. G. \& J. Simpson. 1965. Pheromone of the honeybee (Apis mellifera L.). An olfactory pheromone from the Koschewnikow gland of the queen. Scientific Studies of Uviversity Libice, Czechoslovakia 4: 33-36.

Cassier, P. \& Y. Lensky. 1995. Ultrastructure of wax gland complex and secretion of bees wax in the worker honey bee Apis mellifera L. Apidologie 26: 17-26.

Cavalcante, V. M.; V. T. Paes de Oliveira. \& C. Cruz-Landim. 2000. Comparative study of wax glands in four Meliponini bees (Hymenoptera, Apidae) producing different quantities of wax. Iheringia Série Zoologia 89: 193-198.

Chapman, R. F. 1998. The insects: Structure and function. New York, Elsevier, 918p.

Cruz-Landim, C. 1963. Evolution of the wax and scent glands in the Apidae (Hymenoptera, Apidae). Journal of New York Entomology Society 71: 2-13.

Cruz-Landim, C. 1967. Estudo comparativo de algumas glândulas das abelhas (Hymenoptera, Apoidea) e respectivas implicações evolutivas. Arquivos de Zoologia 15: 177-290.

Cruz-Landim, C.; M. C. Höfling. \& V. L. Imperatriz-Fonseca. 1980a. Tergal and mandibular glands in queens of Paratrigona subnuda (Moure) (Hymenoptera, Apidae). Morphology and associated behaviour. Naturalia 5: 121-133.

Cruz-Landim, C.; S. M. F. Santos \& M. C. Höfling. 1980b. Sex determination in bees. XV. Identification of queens of Melipona quadrifasciata anthidioides (Apidae) with the worker phenotype by a study of the tergal glands. Revista Brasileira de Genética 3: 295-302.

Cruz-Landim, C. \& M. H. V. B. Mota. 1990. Occurrence of tegumentary glands in stingless bees. (Hymenoptera, Apidae, Meliponinae), p.587-588. In: G. K. Veeresh; B. Mallik \& C. A. Viraktamath (eds.). Social insects and the environment. New Delhi, Oxford \& IBH Publishing Co, 765p.

Cruz-Landim, C. 1992. Glândulas exócrinas presentes nos adultos das 
abelhas sociais. Encontro brasileiro sobre biologia de abelhas e outros insetos. Naturalia n. especial: 79-84.

Cruz-Landim, C. \& M. H. V. B. Mota. 1993. Differences between the female castes and males of Scaptotrigona postica depilis (Hymenoptera, Apidae, Meliponinae) in the occurrence and ultrastructure of tegumentary exocrine glands. Naturalia 18: 173187.

Cruz-Landim, C. 1996. Glândulas tegumentares abdominais de abelhasOcorrência e ultra-estrutura. Anais do Encontro sobre Abelhas 2: $67-77$.

Cruz-Landim, C. 1998. Desenvolvimento pós-embrionário das glândulas tegumentares do abdomem das abelhas (Hymenoptera, Apoidea). Anais do Encontro sobre abelhas 3: 109-113.

Cruz-Landim, C. \& R. D. Reginato. 1999. Preliminar report on the presence of tegumentar glands in the thorax of Meliponinae bees (Hymenoptera, Apidae). Revista Brasileira de Biologia 59: $167-172$.

Cunha, M. A.; C. Cruz-Landim \& L. A. O. Campos. 1990. Ocorrência e morfologia das glândulas tegumentares de algumas espécies de abelhas (Hymenoptera, Apoidea). Naturalia 15: 209-217.

Dreyling, L. 1903. Über die waschsbereitenden organe der honigibiene. Zoologischer Anzeiger 26: 710-715.

Espelie, K. E.; V. M. Butz \& A. Dietz. 1990. Dexydecanoate: A major component of the tergite glands of honeybee queens (Apis mellifera L.). Journal of Apicultural Research 29: 15-19.

Gary, N. E. 1963. Observations of mating behaviour in the honeybee. Journal of Apicutural research 2: 3-13.

Ghent, R. \& N. E. Gary. 1962. A chemical alarm releaser in honeybee stings (Apis mellifera L.). Psyche 69: 1-6.

Guerino, A. C. \& C. Cruz-Landim. 1999. A new type of sternal gland present in Oxaea flavescens (Hymenoptera, Oxaeinae): location and histology. Cytobios 97: 71-77.

Guerino, A. C. \& C. Cruz-Landim. 2002. Ultra-estrutura de glândulas abdominais em Oxaea flavescens (Hymenoptera, Andrenidae, Oxaeinae). Iheringia, Série Zoologia 92: 37-45.

Guerino, A. C. \& V. T. Paes de Oliveira. 2002. Glândulas tegumentares de abdômen, p. 111-126. In: C. Cruz-Landim \& F. C. Abdalla (eds.). Glândulas exócrinas das abelhas. Ribeirão Preto, FUNPEC-Editora, $\mathrm{xi}+181 \mathrm{p}$.

Guerino, A. C. \& C. Cruz-Landim. 2003a. Ocorrência e morfologia de glândulas tegumentares no abdome de algumas abelhas (Hymenoptera: Apidae): Um estudo comparado. Neotropical Entomology 32(2): 261-267.

Guerino, A. C. \& C. Cruz-Landim. 2003b. Glândulas tegumentares do abdômen de Bombus morio (Hymenoptera, Apidae, Bombina), p.141-149. In: G. A. R. Melo \& I. Alves-dos-Santos (eds.). Apoidea Neotropica: Homenagem aos 90 anos de Jesus Santiago Moure. Criciúma, Editora UNESC, xvi +320 p.

Jacob, W. 1924. Das duftorgan von Apis mellifera und ähnliche Hautdrüsenorgane um solitarer Apiden. Z. Morphol. Oekol. Tiere 3: $1-80$.

Maschwitz, V. M. 1964. Alarm substance and behaviour in social Hymenoptera. Nature 204: 224-237.

Mcindoo, N. E. 1914. The scent-producing organ of honeybee. Proceedings of Academy of Nature Science 66: 542-555.

Mota, M. H. V. B. \& C. Cruz-Landim. 1988. Ocorrência e morfometria de glândulas tegumentares abdominais em Apis mellifera (Hymenoptera, Apidae). Revista Brasileira de Zoologia 5: 119154.

Noirot, C. \& A. Quennedey. 1974. A Fine structure of insect epidermal glands. Annual Review of Entomology 19: 61-80.

Noirot, C. \& A. Quennedey. 1991. Glands, gland cells, glandular units: Some comments on terminology and classification. Annals of Society Entomologic of France 27: 123-128.

Paes de Oliveira, V. T. \& C. Cruz-Landim. 2001. Experimental control of the effect of extra doses of juvenile hormone on bee development: The case of the wax glands of Apis mellifera (Hymenoptera: Apidae). Sociobiology 38: 513-521.

Ramos, K. S.; C. G. Santos; C. Schlindwein \& B. Blochtein. 2004 Tegumentar glands associated to foveae in the second metasomal tergum of Panurgillus Moure (Apoidea, Andrenidae, Panurginae). Revista Brasileira de Entomologia 48: 163-167.

Salles, H. C. \& C. Cruz-Landim. Levantamento das glândulas presentes em Camargoia nordestina Moure, 1989 (Hymenoptera, Apidae, Meliponinae). Revista Brasileira de Entomologia 41: 297302.

Santos, C. G.; J. M. Oliveira; K. S. Ramos \& B. Blochtein. 2004. Plasticidade de glândulas tegumentares abdominais em Monoeca xanthopyga Harter-Marques, Cunha \& Moure (Hymenoptera, Apidae, Tapinotaspidini). Revista Brasileira de Entomologia 48: $221-225$

Silveira, F. A.; G. A. R. Melo \& E. A. B. Almeida. 2002. Abelhas brasileiras: Sistemática e identificação. Belo Horizonte, 253p.

Velthuis, H. H. W. 1970. Queen substance from the abdomen of the honey bee queen. Physiologie 70: 210-222.

Wilson, E. 1963. Pheromones. Science American 208: 100-114.

Yussef, N. N. 1975. Fine structure of the intersegmental membrane glands of the sixth abdominal sternum of female Nomia melanderi (Hymenoptera, Apoidea). Journal of Morphology 146: $307-$ 324.

Recebido em 02.IX.2004; aceito em 04.VIII.2005 